\title{
TMPO-ASI Regulates the Aggressiveness-Associated Traits of Nasopharyngeal Carcinoma Cells Through Sponging miR-320a
}

This article was published in the following Dove Press journal: Cancer Management and Research

\author{
Biao Xing ${ }^{1, *}$ \\ Xiao-Feng Qiao ${ }^{2, *}$ \\ Yan-Hua Qiu ${ }^{3}$ \\ $\mathrm{X}$ in $\mathrm{Li}^{4,5}$
}

'Department of Otolaryngology, Cangzhou Central Hospital, Cangzhou, Hebei, People's Republic of China; ${ }^{2}$ Department of Otorhinolaryngology, Shanxi Provincial People's Hospital Affiliated to Shanxi Medical University, Taiyuan, People's Republic of China; ${ }^{3}$ Department of Otolaryngology, The First People's Hospital of Linhai City, Linhai, Zhejiang, People's Republic of China; ${ }^{4}$ Department of Otorhinolaryngology, Beijing Tsinghua Changgung Hospital Affiliated to Tsinghua University, Beijing, People's Republic of China; ${ }^{5}$ School of Clinical Medicine, Tsinghua University, Beijing, People's Republic of China

*These authors contributed equally to this work
Correspondence: Xin Li

Department of Otorhinolaryngology, Beijing Tsinghua Changgung Hospital Affiliated to Tsinghua University, Beijing, People's Republic of China

Email lixinlx8@tom.com
Background: Previous evidence demonstrates that the long non-coding RNA (lncRNA) TMPO antisense RNA 1 (TMPO-AS1) is involved in the aggressiveness of several cancers. Nevertheless, its functions in nasopharyngeal carcinoma (NPC) are unclear.

Methods: qRT-PCR was used to evaluate the levels of TMPO-AS1 and miR-320a in NPC tissues. Furthermore, the growth and invasiveness of NPC cells were evaluated by colony formation and Transwell assays. The protein expression ofSRY-Box Transcription Factor 4 (SOX4) was observed by Western blotting and immunohistochemistry. Bioinformatic prediction and luciferase reporter assays were used to explore the interaction between miR-320a and TMPO-AS1. The transplanted model was employed to disclose the interference of TMPO-AS1 in the tumor growth of NPC cells in vivo.

Results: We found that TMPO-AS1 was distinctly upregulated in NPC. Downregulation of TMPO-AS1 restrained aggressiveness-associated traits in NPC cells. Nevertheless, upregulation of TMPO-AS1 yielded the opposite results. Further studies revealed that lncRNA TMPO-AS1 acts as a "sponge" for miR-320a, resulting in increased levels of SOX4 in NPC cells. Finally, TMPO-AS1 silencing suppressed tumor growth of NPC cells in vivo.

Conclusion: Collectively, these results reveal the presence of a novel TMPO-AS1/miR$320 \mathrm{a} / \mathrm{SOX} 4$ pathway associated with NPC progression, suggesting that lncRNA TMPO-AS1 may be a potential therapeutic target for NPC.

Keywords: TMPO-AS1, nasopharyngeal carcinoma, invasion, SOX4

\section{Background}

Etiological factors for nasopharyngeal carcinoma (NPC) include genetic factors, environmental factors, and Epstein-Barr virus (EBV) infection. Currently, radiotherapy and chemotherapy remain the standard treatments for late-stage NPC. ${ }^{1,2}$ Despite the improvement of radiotherapy and chemotherapy in combating NPC, recurrence and metastasis are frequently observed in patients. ${ }^{3}$ Hence, promising biomarkers and curative options are urgently warranted to improve the clinical prognosis in patients with NPC.

Recently, long noncoding RNAs (IncRNAs) have been found to be involved in a variety of physiological and pathological processes, especially in cancer. ${ }^{4}$ LncRNAs are transcripts with more than 200 nucleotides; they have no proteincoding potential. LncRNAs are frequently dysregulated in cancer and are involved in the progression and metastasis of multiple malignancies. ${ }^{5,6}$ Recently, TMPO-AS1 has been reported as an oncogene in several cancer types. TMPO-AS1 promotes the 
progression of cervical cancer cells by upregulating RasRelated Protein Rab-14 (RAB14) via sponging miR-577. ${ }^{7}$ TMPO-AS1 also promotes cervical cancer cells' proliferation, migration, and invasion by regulating the miR-1433p-Zinc Finger E-Box Binding Homeobox 1 (ZEB1) axis. ${ }^{8}$ In hepatocellular carcinoma, TMPO-AS1 promotes cancer cells' proliferation, migration, and invasion through sponging miR-329-3p to stimulate the Forkhead Box K1 (FOXK1)-mediated serine/threonine kinase 1-mammalian target of rapamycin (AKT-mTOR) signaling pathway. ${ }^{9}$ Several studies suggest that lncRNAs may function as competing endogenous RNAs (ceRNAs) to regulate the biological function or expression of miRNAs. ${ }^{10}$ LncRNA TMPO-AS1 serves as a ceRNA to promote osteosarcoma tumorigenesis by regulating the miR-199a-5p-Wnt Family Member 7B (WNT7B) axis. ${ }^{11}$ Although TMPO-AS1 has also been reported to be involved in several cancers, the underlying regulatory mechanism of TMPO-AS1 in NPC has not been established.

A majority of miRNAs serve as oncogenes or tumor suppressors in a tissue-specific manner, and dysregulation of miRNAs is closely linked to carcinoma progression. ${ }^{12}$ Chemically, antisense oligonucleotides efficiently and specifically inhibit miRNA modified function. In contrast, miRNA mimics could functionally replenish lost miRNA expression in specific diseases, highlighting the potential for developing novel therapeutic methods. ${ }^{13,14}$ Mounting evidence has uncovered that miR-320a can suppress tumor growth in several cancers. miR-320a serves as a negative regulator in the progression of gastric cancer by targeting RAB14. ${ }^{15}$ miR-320a inhibits non-small cell lung cancer cells metastasis via regulating the PI3K/AKT pathway. ${ }^{16}$ Nevertheless, the association of miR-320a with NPC has not yet been explored.

In the present study, we disclosed that lncRNA TMPOAS1 was significantly overexpressed in NPC. TMPO-AS1 knockdown inhibited NPC cells' growth and suppressed the invasive ability of these cells. Further study revealed that TMPO-AS1 regulated the aggressiveness-associated traits of NPC cells by sponging miR-320a. Collectively, our results provide novel insights into the functions of the TMPO-AS1-miR-320a axis in NPC.

\section{Materials and Methods}

\section{Nasopharyngeal Carcinoma Specimens}

A total of 45 NPC tissue samples were collected from participants who underwent NPC biopsy at Cangzhou
Central Hospital (Hebei, China) between 2010 and 2018, and 22 normal nasopharynx tissues were collected from patients with chronic nasopharyngitis, at Cangzhou Central Hospital. All pathological slices were reviewed by two experienced pathologists. The inclusion criteria were as follows: NPC diagnosed with an electronic nasopharyngoscope, NPC confirmed histologically by biopsy, no previous treatment for NPC, and no history of any other malignant tumors. The exclusion criteria included recurrent NPC, patients positive for other malignancies and having a history of radiotherapy or chemotherapy. An informed consent was obtained from study participants, and this study was approved by the Ethics Committee of Cangzhou Central Hospital (Hebei, China). The study conformed to the Code of Ethics of the World Medical Association (Declaration of Helsinki) printed in the British Medical Journal (July 18, 1964).

\section{Cell Lines}

Human nasopharyngeal carcinoma cell lines (SUNE-1 and C666-1) were maintained in RPMI-1640 (Thermo Fisher Scientific, Waltham, MA, USA) with 10\% FBS (Thermo Fisher Scientific). Cell lines were achieved from Guangzhou Jennio Biotech Co., Ltd (Guangzhou, China) and were maintained at $37^{\circ} \mathrm{C}$ in $5 \% \mathrm{CO}_{2}$.

\section{Cell Transfection}

miR-320a mimics and negative control (miR-Ctrl), shRNA against TMPO-AS1 (sh-TMPO-AS1) and shRNA negative control (sh-Ctrl) were constructed by GenePharma (Shanghai, China). The human miR-320a mimics sequence was 5'-AAAAGCUGGGUUGAGAGGGCGA-3'. The sequence for miR-Ctrl was 5'-CAGUACUUUUGUGU AGUACAA-3'. The pcDNA3.1 control plasmid (pcDN A3.1-NC) and plasmid for mediating TMPO-AS1 overexpression (pcDNA3.1-TMPO-AS1) were obtained from GenePharma. SUNE-1 and C666-1 cells were transfected with miRNA or pcDNA3.1 by utilizing Lipofectamine 3000 (Thermo Fisher Scientific).

\section{qRT-PCR}

RNAs were isolated using a TRIZOL kit. RNA $(1 \mu \mathrm{g})$ was utilized for cDNA synthesis by using reverse transcriptase PCR. qRT-PCR was employed to measure the level of TMPO-AS1 and SOX4 using SYBR Green qPCR Master Mix (TAKARA, Dalian, China). All-in-One miRNA qRTPCR Detection Kit (GeneCopoeia, Guangzhou, China) was utilized to assess the level of miR-320a. The primer 
Table I Primer Sequence

\begin{tabular}{|l|l|}
\hline Primer & Sequence (5'-3') \\
\hline SOX4 Forward & AGCGACAAGATCCCTTTCATTC \\
SOX4 Reverse & CGTTGCCGGACTTCACCTT \\
miR-320a Forward & AAGGGATCGCGGGCG \\
miR-320a Reverse & TGCGTGTCGTGGAGTC \\
TMPO-ASI Forward & GGGGTAGCAGCACAGAAAT \\
TMPO-ASI Reverse & TCCAGTGCGTGTCGTGGA \\
U6 Forward & GCTTCGGCAGCACATATACTAAAAT \\
U6 Reverse & CGCTTCACGAATTTGCGTGTCAT \\
GAPDH Forward & CAACGAATTTGGCTACAGCA \\
GAPDH Reverse & AGGGGTCTACATGGCAACTG \\
\hline
\end{tabular}

sequence in PCR is shown in Table 1. GAPDH and U6 were used for normalization. The expression of RNA was normalized against GAPDH using the $2^{-\Delta \Delta \mathrm{Ct}}$ method.

\section{Cell Proliferation}

C666-1 or SUNE1 were cultured in 96-well plates $\left(1 \times 10^{4}\right)$. After reached $80 \%$ confluence, $10 \mu \mathrm{L}$ of CCK- 8 solution was added to each well at $24,48,72$ or 96 hours, respectively. After 4 hours, cell supernatant was removed and DMSO $(200 \mu \mathrm{L})$ was plated into the plate. The optical density (OD) value was assessed at $450 \mathrm{~nm}$.

\section{5-Ethynyl-2'-Deoxyuridine (EdU) Incorporation Assay}

Transfected cells were seeded into 96-well plates at a density of $1 \times 10^{4}$ cells/well. The EdU incorporation assay kit (RiboBio, Guangzhou, China) was used to evaluate cell proliferation. A fluorescence microscope (Nikon, Japan) was used to obtain images.

\section{Colony Formation Assay}

After transfection, C666-1 or SUNE1 cells (1000 cells/ well) were plated into six-well plates. After 14 days, colonies were fixed using methanol and dyed with $0.1 \%$ crystal violet (Beyotime Biotechnology, Jiangsu, China).

\section{Invasion Assay}

The membrane of Transwell with 8 - $\mu \mathrm{m}$ polycarbonate nucleopore filter (Corning Costar, Corning, NY, USA) was coated with $40 \mu \mathrm{L}$ of Matrigel (BD, Shanghai, China) to form a basement membrane. After transfection, $200 \mu \mathrm{L}$ of C666-1 or SUNE1 cells was plated into the Transwell unit. After $18 \mathrm{~h}$, invasive cells were dyed by crystal violet (Beyotime Biotechnology, Jiangsu, China).

\section{Immunoblotting}

Proteins in cells were extracted using RIPA buffer. Total proteins were separated utilizing $8 \%$ SDS-PAGE and following transferred on the PVDF membrane. After incubated with SOX4 or $\beta$-tubulin antibody (1:1000, Santa Cruz Biotechnology, CA, USA) at $4^{\circ} \mathrm{C}$ overnight, membrane was incubated with secondary antibody (1:10000, Bioworld, Jiangsu, China) for 2 hours. The target band was measured using ECL reagent (Millipore, Braunschweig, Germany).

\section{Luciferase Reporter Assay}

SOX4 3'-UTR (UACACAAUCUGCCUUAGCUUUAA) or TMPO-AS1 3'-UTR (CCCAGCTTGAGAAGCAGC TTTC) harboring binding site among miR-320a was cloned into pmirGLO luciferase reporter (Promega, Madison, WI, USA) to generate pmirGLO-SOX4-wt or pmirGLO-TMPOAS1-wt, respectively. The mutated SOX4 (UACACAAUC UGCCUUUCUCCGUA) or TMPO-AS1 binding sequence (CCCAGCTTGAGAAGAGCGAAAC) was constructed using the QuikChange Site-Directed Mutagenesis Kit (Stratagene, USA) to construct pmirGLO-SOX4-mut and pmirGLO-TMPO-AS1. SUNE1 or C666-1 cell was cotransfected with miR-320a mimics and pmirGLO report. After $24 \mathrm{~h}$, the luciferase activity was measured by Luciferase Reporter Assay Kit (Promega).

\section{Mouse Experiment}

BALB/c nude mice were subcutaneously inoculated with shCtrl or sh-TMPO-AS1 transfected SUNE1 $\left(2 \times 10^{6}\right)$ cells. Tumor volume $=0.5 \times$ length $\times$ width $^{2}$. The width $(\mathrm{mm})$ and length $(\mathrm{mm})$ of xenograft tumor were measured each week. In the rescue assay, SUNE1 cells were transfected with miRCtrl or cotransfected with miR-320a with pcDNA3.1-TMPO-AS1. BALB/c nude mice were subcutaneously inoculated with transfected SUNE1 cells. After 5 weeks, mice were sacrificed, and xenograft tumor was subjected for immunohistochemistry analysis using SOX4 antibody. The animal experiment was approved by Cangzhou Central Hospital. The study was authorized by the Ethics Committee of Cangzhou Central Hospital (Hebei, China) and was conducted in accordance with Institutional Guidelines and the Guide for the Care and Use of Laboratory Animals (NIH publication no. 85-23, revised 1996).

\section{Data Analysis}

SPSS statistics 19.0 software was used to analyze and process the experimental data, GraphPad Prism 8.0 software (San 
Diego, CA, USA) was employed to generate the histograms of various kinds shown. Data are presented as mean $\pm \mathrm{SD}$. Difference was calculated using Student's $t$-test or one-way ANOVA followed by Dunnett's test. Spearman correlation was used to analyze the correlation between TMPO-AS1 and miR-320a. As for categorical data, Chi-square test was utilized to analyze the correlation between TMPO-AS1 and clinicopathological parameters. $P<0.05$ was statistical difference.

\section{Results}

\section{TMPO-ASI is Upregulated in NPC}

First, we assessed the expression of TMPO-AS1 in 45 cancer samples and 22 normal nasopharyngeal tissue samples obtained from patients who were finally diagnosed with chronic nasopharyngitis, using qRT-PCR. The level of TMPO-AS1 was significantly higher in NPC tissue than in normal tissue (Figure 1A). The median value of TMPO-AS1 expression was 0.56. Clinicopathologically, a higher level of TMPO-AS1 was associated with metastasis and advanced clinical stage in patients with NPC (Table 2). However, no significant correlation was observed in between the expression of TMPO-AS1 and gender, age, and smoking history
$(P>0.05)$ in NPC tissues. C666-1 or SUNE1 cells were transfected with sh-Ctrl or sh-TMPO-AS1, and it was observed that TMPO-AS1 knockdown distinctly decreased TMPOAS1 levels (Figure 1B). Following transfection of sh-TMPOAS1, the proliferation of C666-1 and SUNE1 cells was markedly reduced as seen in the CCK-8 (Figure 1C and D) and EdU assay (Figure 1E). All the data indicated that TMPOAS1 knockdown inhibited the growth of NPC cells in vitro.

\section{TMPO-ASI Silencing Impedes NPC Cell Growth in vitro}

Next, a colony formation test was used to estimate the interference of TMPO-AS1 on NPC cell growth. As shown in Figure 2A, transfection of sh-TMPO-AS1 largely impaired the colony formation abilities of both SUNE1 and C666-1 cells. In addition, the Transwell assay indicated a remarkable decrease in the invasiveness of NPC cells after introduction of sh-TMPO-AS1 (Figure 2B). On the contrary, the upregulation of TMPO-AS1 using pcDNA3.1-TMPO-AS1 enhanced NPC cells' growth and invasion (Figure 2C-E). Collectively, the data illustrated that TMPO-AS1 knockdown restrained the growth rate and invasive properties of NPC cells in vitro.

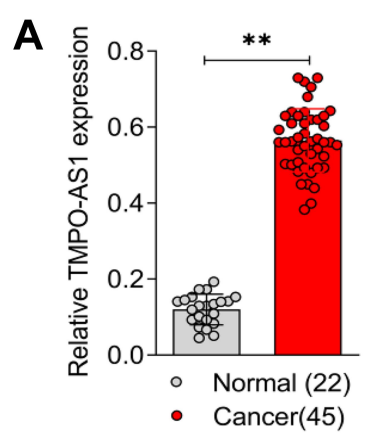

B

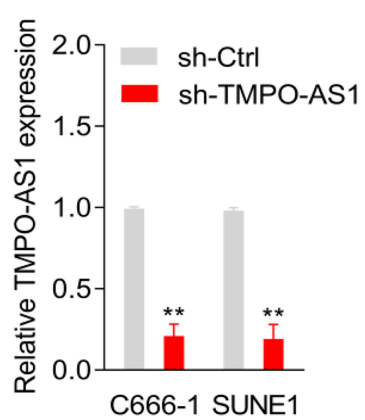

C

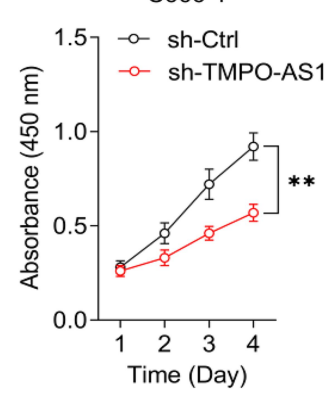

D

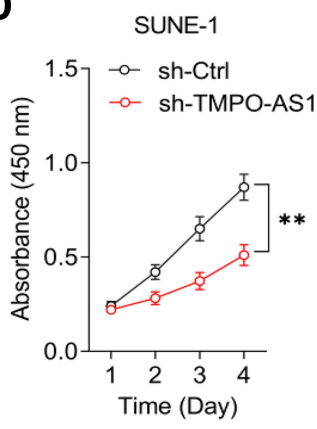

E
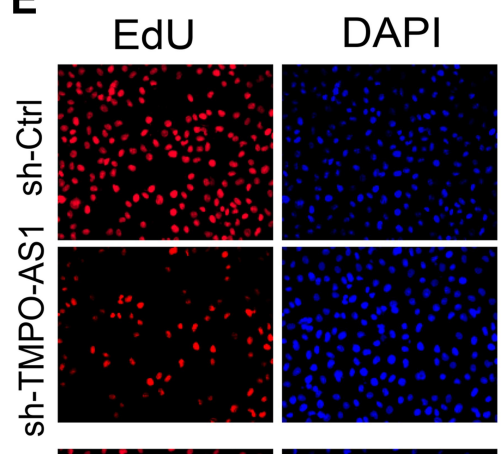

Merged

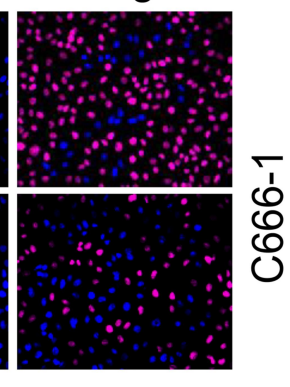

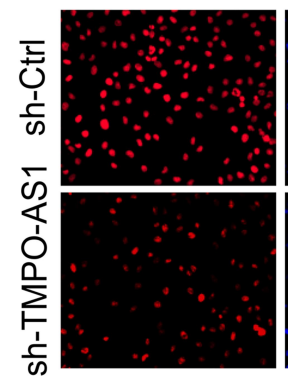
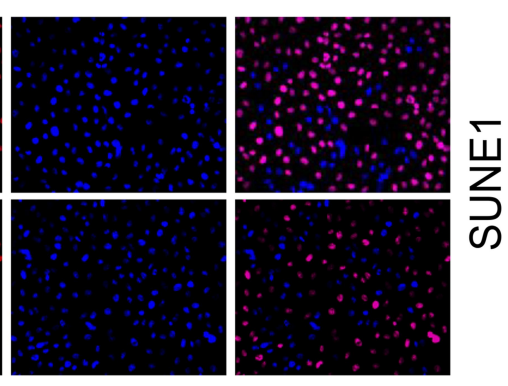

Figure I TMPO-ASI is upregulated in nasopharyngeal carcinoma. (A) TMPO-ASI expressions in 45 nasopharyngeal carcinoma and normal tissues were assessed by qRTPCR. ${ }^{*} * P<0.01$ compared with normal. (B) C666-I and SUNEI cell were transfected with sh-Ctrl or sh-TMPO-ASI. The expression levels of TMPO-ASI in C666-I and SUNEI cell were detected using qRT-PCR analysis. (C and D) CCK-8 assays were performed with C666-I and SUNEI cell after transfection with sh-TMPO-ASI or sh-Ctrl. $* * P<0.01$ compared with sh-Ctrl. (E) The proliferation of C666-I and SUNEI was evaluated by EdU staining assay. 
Table 2 Correlation Between TMPO-ASI Expression and Clinical Characteristics of Patients with Nasopharyngeal Carcinoma

\begin{tabular}{|c|c|c|c|c|c|}
\hline \multirow[t]{2}{*}{ Variables } & \multirow[t]{2}{*}{ Case } & \multicolumn{2}{|c|}{ TMPO-ASI } & \multirow[t]{2}{*}{$\chi^{2}$} & \multirow[t]{2}{*}{$P$} \\
\hline & & $\begin{array}{l}\text { High } \\
\text { (n) }\end{array}$ & $\begin{array}{l}\text { Low } \\
\text { (n) }\end{array}$ & & \\
\hline Age (years) & & & & 0.204 & 0.32 \\
\hline$<50$ & 24 & 15 & 9 & & \\
\hline$>50$ & 21 & 13 & 8 & & \\
\hline Gender & & & & 5.002 & 0.103 \\
\hline Male & 19 & 12 & 7 & & \\
\hline Female & 26 & 16 & 10 & & \\
\hline Histological type & & & & 1.860 & 0.625 \\
\hline Non-keratinized type & 29 & 18 & II & & \\
\hline Keratinized type & 16 & 10 & 6 & & \\
\hline Clinical stage & & & & 9.703 & 0.012 \\
\hline I-II & 15 & 3 & 12 & & \\
\hline III-IV & 30 & 25 & 5 & & \\
\hline Smoking history & & & & 2.518 & 0.116 \\
\hline Yes & 25 & 14 & 11 & & \\
\hline No & 20 & 14 & 6 & & \\
\hline EBV status & & & & 0.018 & 0.407 \\
\hline Positive & 34 & 20 & 14 & & \\
\hline Negative & 11 & 8 & 3 & & \\
\hline $\begin{array}{l}\text { Lymph node } \\
\text { metastasis }\end{array}$ & & & & 0.620 & 0.018 \\
\hline No & 23 & 8 & 15 & & \\
\hline Yes & 22 & 20 & 2 & & \\
\hline
\end{tabular}

\section{TMPO-ASI Acts as a "Sponge" for miR- $320 \mathrm{a}$}

The starBASE v3.0 tool was used to predict the targeting relationship between miRNAs and TMPO-AS1. As shown in Figure 3A, TMPO-AS1 harbors conjectural binding sites of miR-320a. To determine whether IncRNA TMPOAS1 interacted with miR-320a, a luciferase reporter test was carried out. As depicted in Figure 3B, miR-320a transfection markedly reduced the luciferase activity in NPC cells transfected with the pmirGLO reportercarrying TMPO-AS1-wt but displayed no significant inhibitory effect on SUNE1 and C666-1 cells transfected with a reporter-carrying TMPO-AS1-mut. Moreover, we found that the depletion of TMPO-AS1 increased the expression of miR-320a, whereas TMPO-AS1 overexpression reduced the expression of miR-320a in NPC cells (Figure 3C). More importantly, miR-320a was downregulated in
NPC, compared to the case in normal tissues (Figure 3D). Spearman correlation analysis revealed that the miR-320a level was inversely associated with TMPO-AS levels in NPC tissues (Figure 3E). To explore the function of miR320a in NPC, miR-320a mimics were transfected into SUNE1 and C666-1 cells and the transfection efficiency was confirmed by qRT-PCR analysis (Figure 3F). The following colony formation assay also showed that the growth of SUNE1 and C666-1 cells was dramatically decreased in the miR-320a-transfected group (Figure $3 \mathrm{G})$. As expected, the invasion of NPC cells was also inhibited by miR-320a (Figure $3 \mathrm{H}$ ). All study findings proved that miR-320a inhibited the aggressiveness of NPC cells.

\section{TMPO-ASI Upregulates SOX4 Expression via Inhibition of miR-320a}

Using the TargetScan, picTar, RNA22, PITA, and miRanda databases, a total of six genes (E2F7, SOX4, NRP1, ZFP91, AP3M1, and SERBP1) were obtained from five bioinformatics analyses (Figure 4A). We found that the mRNA level of SOX4 was reduced by miR-320a in NPC cells and thus SOX4 was a potential target of miR-320a for further observation (Figure 4B). The luciferase reporter gene experiment was used to validate whether miR-320a binds to the SOX4 3'-UTR. The results implied that the luciferase activity in pmirGLO reporter-carrying SOX4-wt-transfected cells was decreased by TMPO-AS1, but that of the pmirGLOSOX4 3'-UTR mut was not decreased by miR-320a (Figure 4C). Additionally, we found that the expression of SOX4 was higher in NPC tissues compared to normal tissues, using immunohistochemistry (Figure 4D). Furthermore, the protein levels of SOX4 were reduced by miR-320a overexpression (Figure 4E) or TMPO-AS1 knockdown (Figure 4F).

\section{TMPO-ASI Regulates the Metastatic-Associated Traits via Sponging miR-320a}

SUNE1 or C666-1 cells were transfected with miR-320a or miR-320a plus pcDNA3.1-TMPO-AS1. The cotransfection efficiency was ascertained using a qRT-PCR assay. As shown in Figure 5A, the level of miR-320a increased by miR-320a mimic transfection was markedly impaired upon TMPO-AS1 transfection. As expected, the protein expression of SOX4 was rescued 


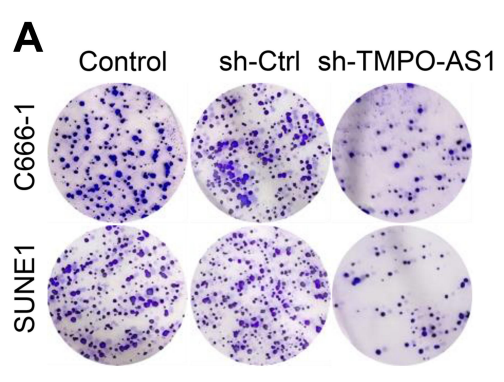

C

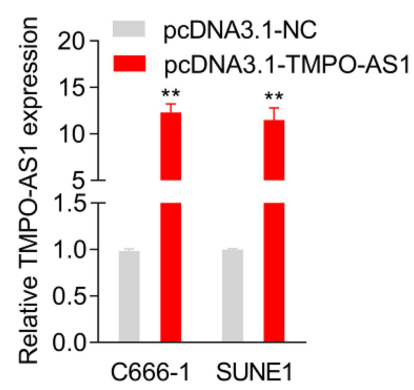

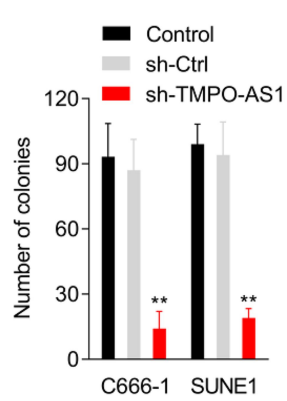
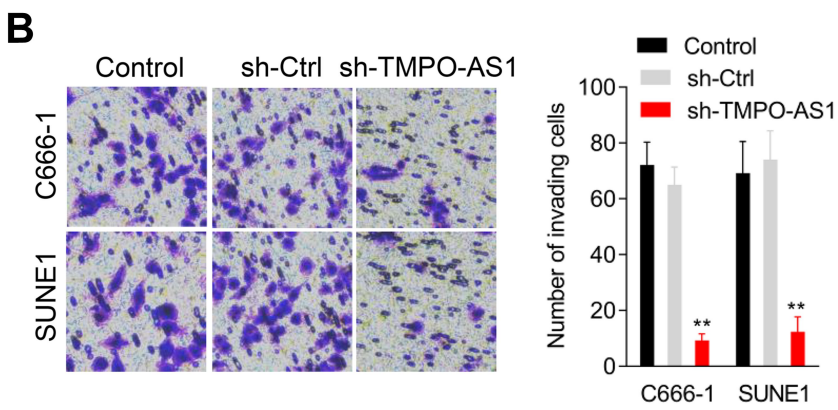

D
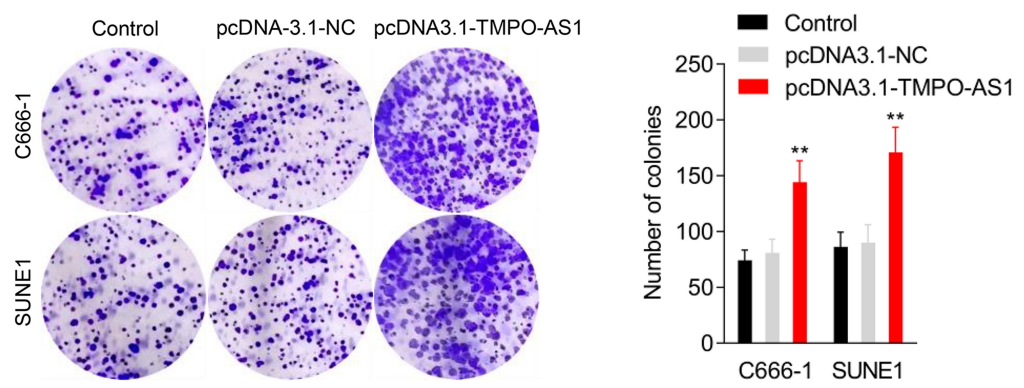
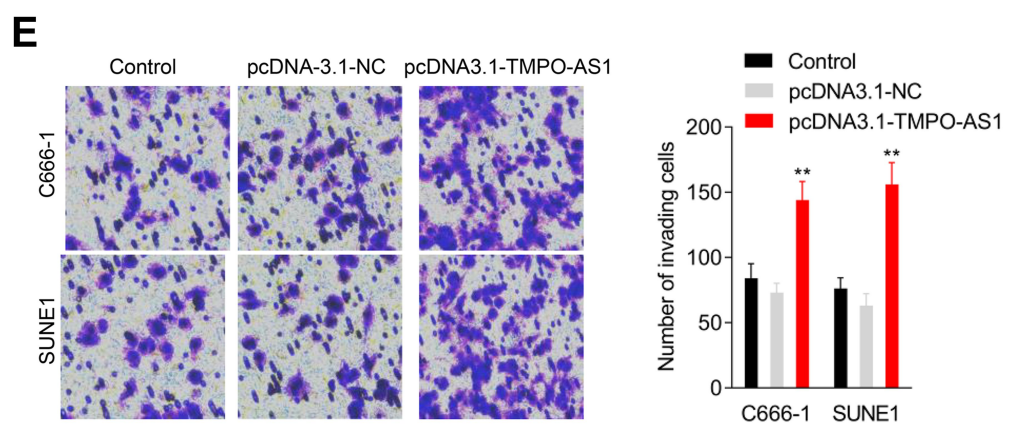

Figure 2 Downregulation of TMPO-ASI inhibits nasopharyngeal carcinoma cell growth in vitro. (A) Colony formation assays were performed with C666-I and SUNEI cell after transfection with sh-TMPO-ASI or sh-Ctrl. (B) Transwell invasion assays were performed with C666-I and SUNEI cell after transfection with sh-TMPO-ASI. **P<0.0I compared with sh-Ctrl. (C) C666-I and SUNEI cell were transfected with pcDNA3.I-TMPO-ASI or its negative control (pcDNA3.I-NC). The expression levels of TMPOASI in C666-I and SUNEI cell were detected using qRT-PCR analysis. (D) Colony formation assays were performed with C666-I and SUNEI cell after transfection with pcDNA3.I-TMPO-ASI or pcDNA3.I-NC. (E) Transwell invasion assays were performed with C666-I and SUNEI cell after transfection with pcDNA3.I-TMPO-ASI.

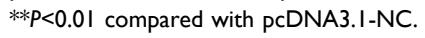

by TMPO-AS1 cotransfection (Figure 5B). The CCK-8 and colony-forming assay indicated that restoration of TMPO-AS1 ameliorated the repressive influence of miR-320a on NPC cells' growth in vitro (Figure 5C and D). Transwell tests showed that restoration of SOX4 terminated the inhibitory effect of miR-320a on the invasion capacity of NPC cells (Figure 5E). All observations suggest that TMPO-AS1 regulates the metastatic-associated traits by sponging miR-320a. A xenograft model of NPC SUNE1 cell was constructed to verify the suppressive impact of TMPO-AS1 knockdown on NPC cells' growth. As displayed in Figure 5F and $\mathrm{G}$, a noteworthy decrease was observed in tumor volume in the sh-TMPO-AS1 group, compared to the case for the sh-Ctrl group. The immunohistochemistry staining assay also depicted a remarkable inhibition in the expression of SOX4, in mice injected with sh-TMPO -AS1-transfected cells (Figure 5H). Moreover, qRT-PCR assay confirmed that sh-TMPO-AS1 increased miR-320a expression (Figure 5I). To ascertain whether TMPO-AS1 aggravated NPC cells' growth in vivo by sponging miR$320 \mathrm{a}$, an in vivo rescue experiment was performed. Nude mice were injected with miR-320a-transfected SUNE1 cells or miR-320a/pcDNA3.1-TMPO-AS1cotransfected SUNE1 cells. As shown in Figure 5J and $\mathrm{K}$, the growth of SNUE1 in vivo was prominently 
A

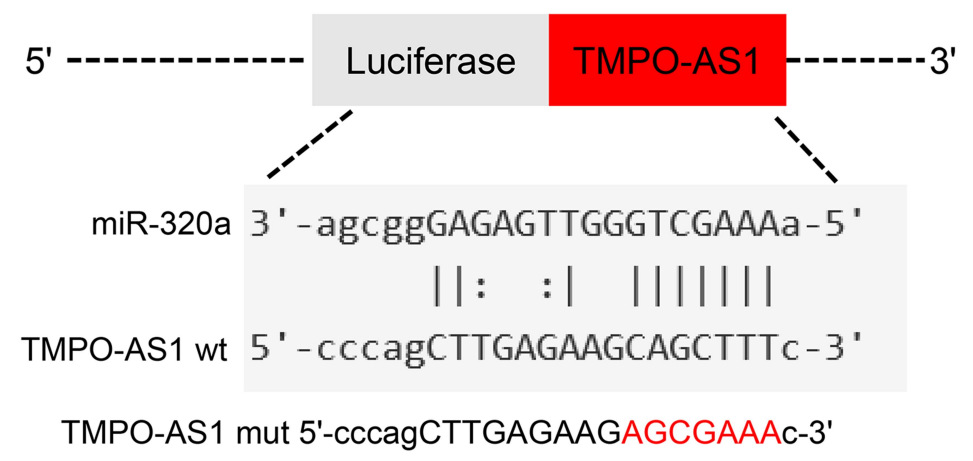

C

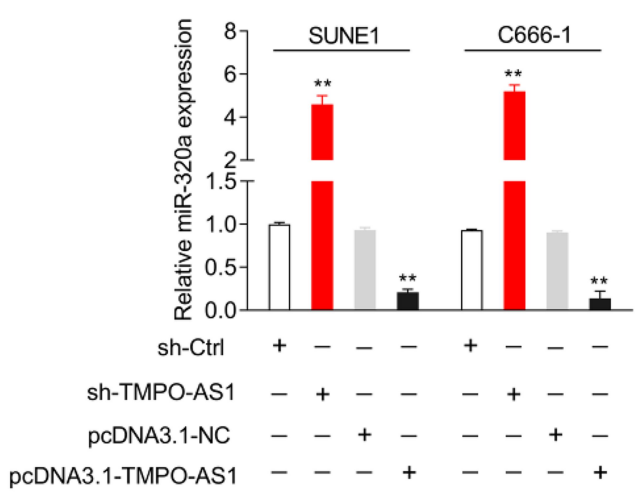

D

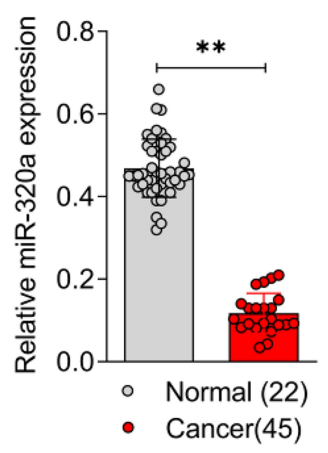

B

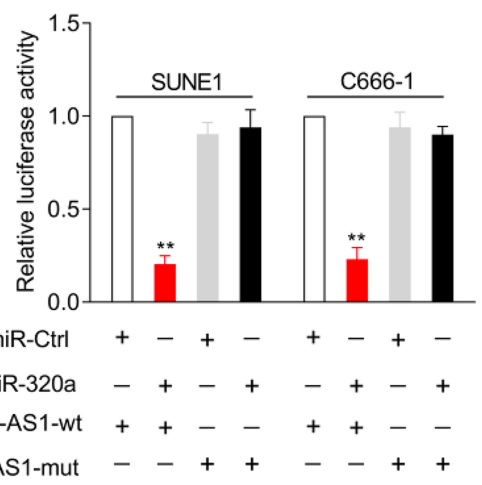

E

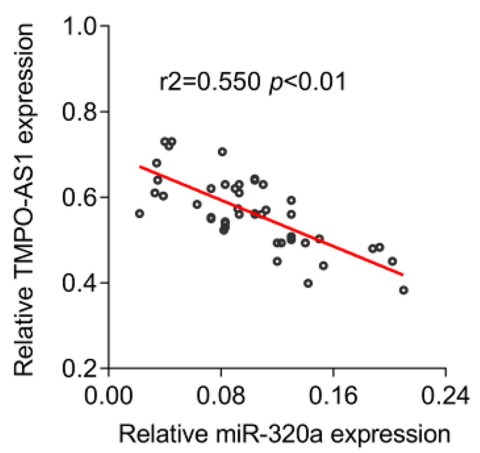

F

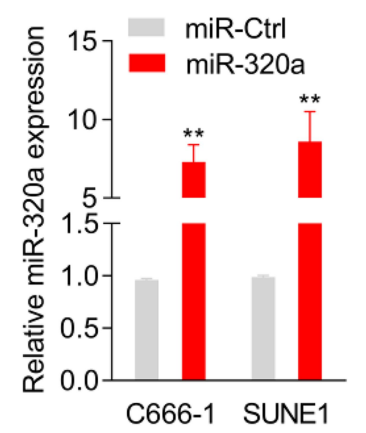

G

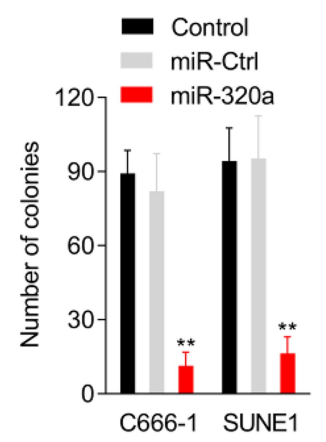

H

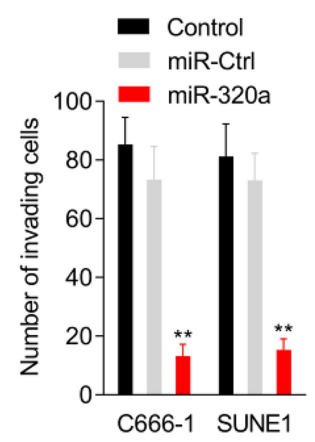

Figure 3 The negative relationship between miR-320a and TMPO-ASI. (A) The prediction targeting site of TMPO-ASI 3'-UTR combined with miR-320a were shown by using starBASE v3.0. (B) C666-I or SUNEI cell was cotransfected with wild-type 3'-UTR (wt) or mutant-type 3'-UTR (mut) reporter and miR-320a mimic. Luciferase activity was measured. (C) C666-I or SUNEI cell was transfected with sh-TMPO-ASI or pcDNA3.I-TMPO-ASI. The expression of miR-320a was detected using qRT-PCR. (D) miR-320a expressions in 45 paired nasopharyngeal carcinoma and normal tissues were assessed by qRT-PCR. (E) Interactions among SOX4 and TMPO-ASI was assessed by Pearson's correlation analysis. (F) C666-I and SUNEI cell was transfected with miR-Ctrl or miR-320a. The level of miR-320a was assessed using qRT-PCR. (G and H) Colony formation and Transwell invasion assays were performed with the transfected cells. $* * P<0.01$ compared with miR-Ctrl.

decreased after miR-320a mimics were transfected into SNUE1 cells. However, the inhibition of SUNE1 cells' growth induced by miR-320a mimics was distinctly counteracted by pcDN3.1-TMPO-AS1. Immunohistochemistry staining assay demonstrated that the suppression of SOX4 expression caused by miR- 320a mimics was rescued by the upregulation of TMPOAS1 (Figure 5L). Furthermore, the upregulation of TMPO-AS1 declined the level of miR-320a in tumor tissue raised by miR-320a mimics transfection (Figure $5 \mathrm{M})$. To summarize the results, TMPO-AS1 silencing suppressed the tumorigenicity of NPC cells in vivo. 
A

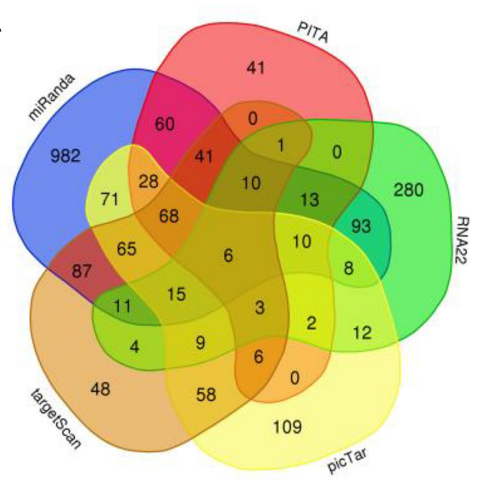

C

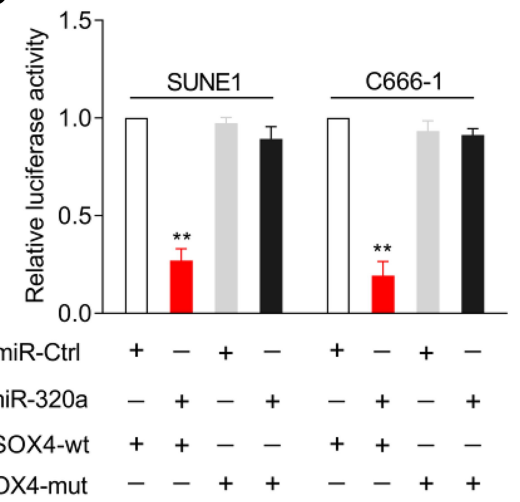

B

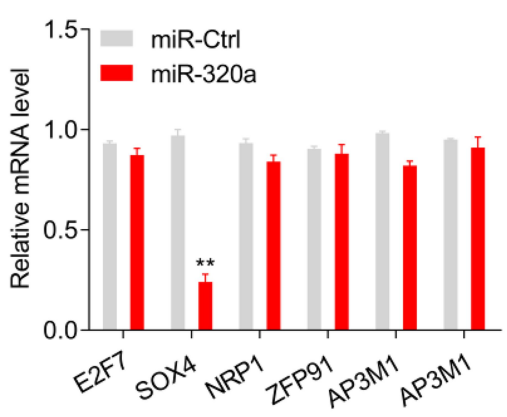

SUNE1

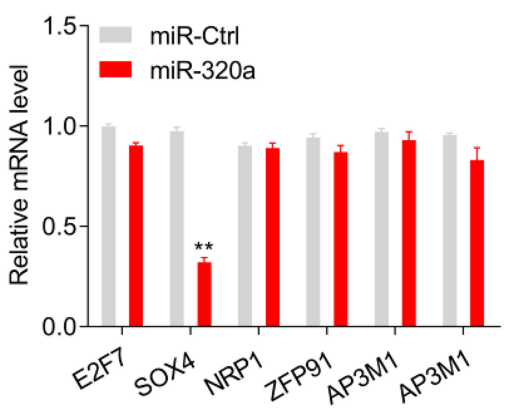

D

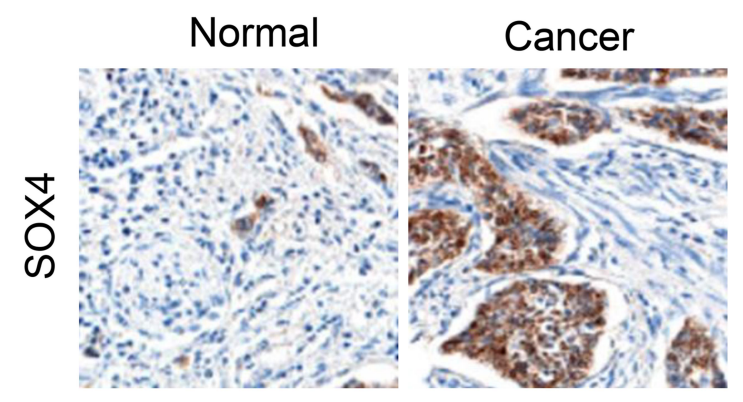

$\mathbf{F}$

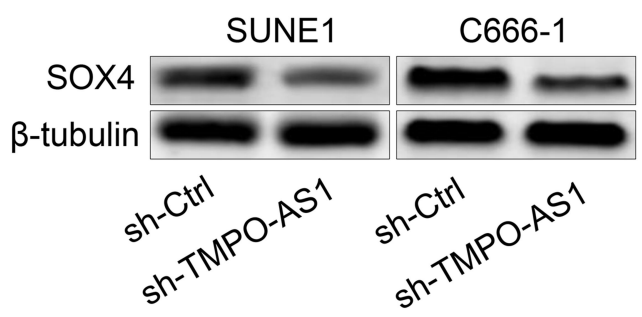

Figure 4 miR-320a directly targets the SOX4 3'-UTR. (A) Venn diagram showing the overlapping target genes of miR-320a from TargetScan, picTar, RNA22, PITA and miRanda databases. (B) qRT-PCR assay was performed to detect the levels of potential genes of miR-320a. (C) C666-I or SUNEI cell was cotransfected with wild-type 3'UTR (wt) or mutant-type 3'-UTR (mut) reporter and miR-320a mimic. Luciferase activity was measured. (D) The expression of SOX4 in nasopharyngeal carcinoma tissue and normal tissue were detected using immunohistochemistry staining. (E) Western blotting were performed to detect the protein expression levels of SOX4 in C666-I and SUNEI cell transfected with miR-Ctrl or miR-320a mimic. (F) Western blotting were performed to detect the protein expression levels of SOX4 in C666-I and SUNEI cell transfected with sh-TMPO-ASI. $* * P<0.01$ compared with miR-Ctrl.

\section{Discussion}

Although radiotherapy is a powerful treatment strategy for early-stage NPC, most NPC cases are predisposed to metastasis at initial diagnosis, which hinders effective treatment and results in frequent recurrence. ${ }^{17}$ Improved comprehension of mechanisms behind NPC could facilitate the improvement of novel metastasis-targeted treatments and improve disease prognosis. ${ }^{18}$ Multiple lines of evidence have verified that dysregulation of lncRNAs affects epigenetic information and may have a significant role in NPC. ${ }^{19,20}$ Consequently, the description of NPC- related lncRNAs and their functions could provide new insights into NPC. ${ }^{21}$

Increasing evidence has confirmed that IncRNA TMPO-AS1 is upregulated in diverse types of cancers, including triple-negative breast cancer, thyroid cancer, and gastric carcinoma. ${ }^{22-24}$ Similarly, in the current study, TMPO-AS1 was discovered to be distinctly upregulated in NPC tissue samples. TMPO-AS1 is reported to act as an essential participant in cancer cells' growth and metastasis. For instance, TMPO-AS1 facilitates the proliferation and metastasis of non-small cell lung cancer cells 

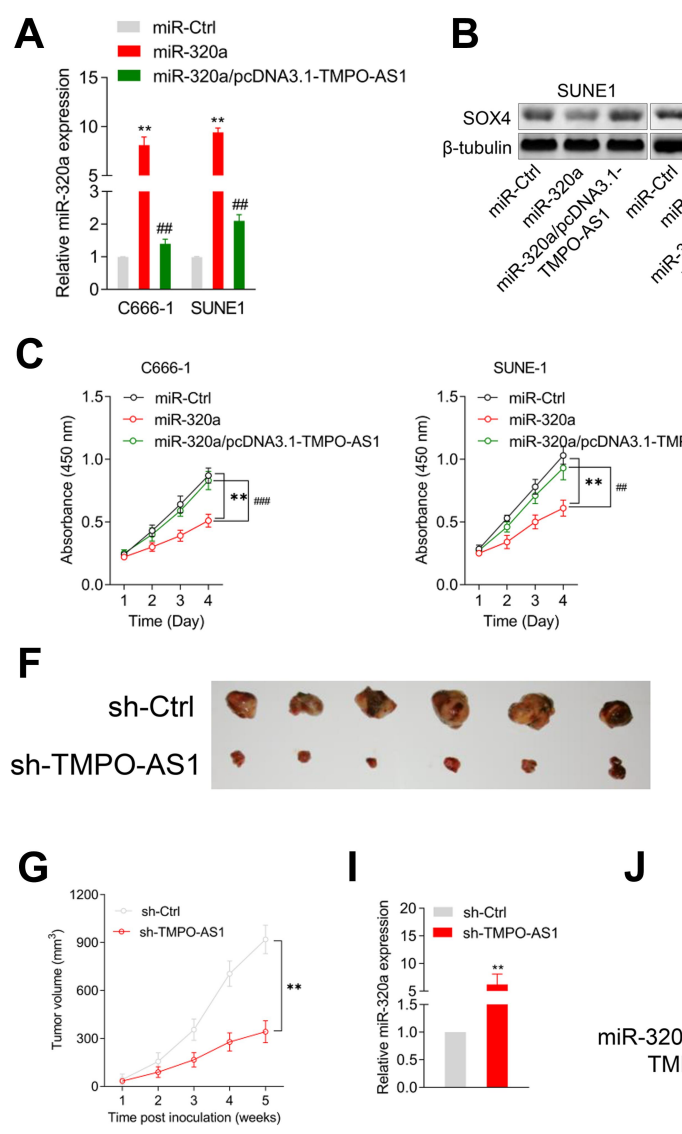
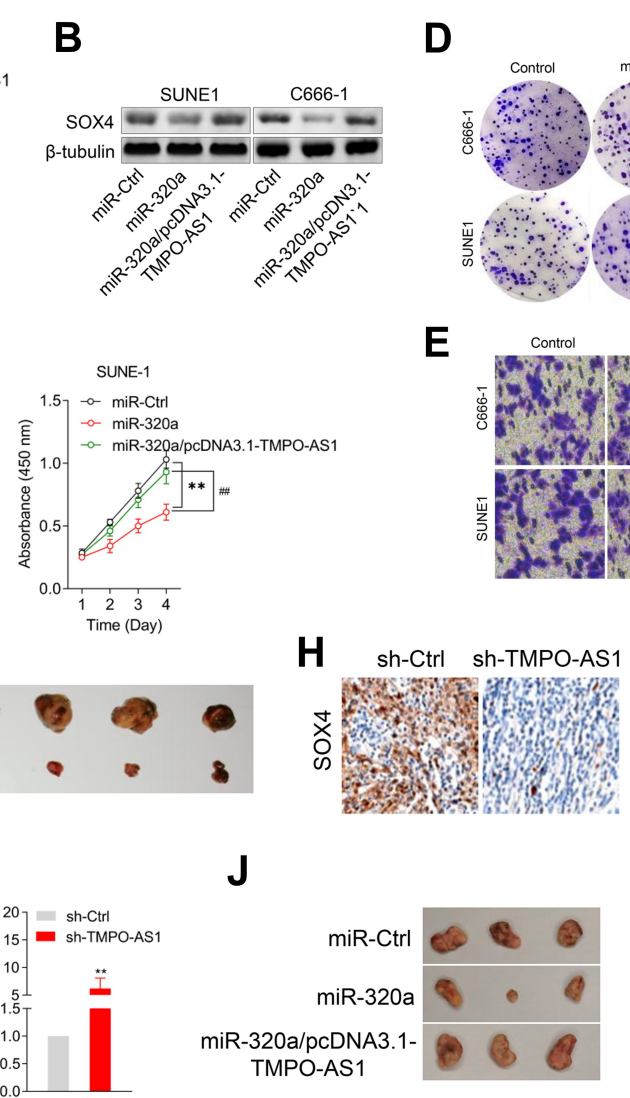
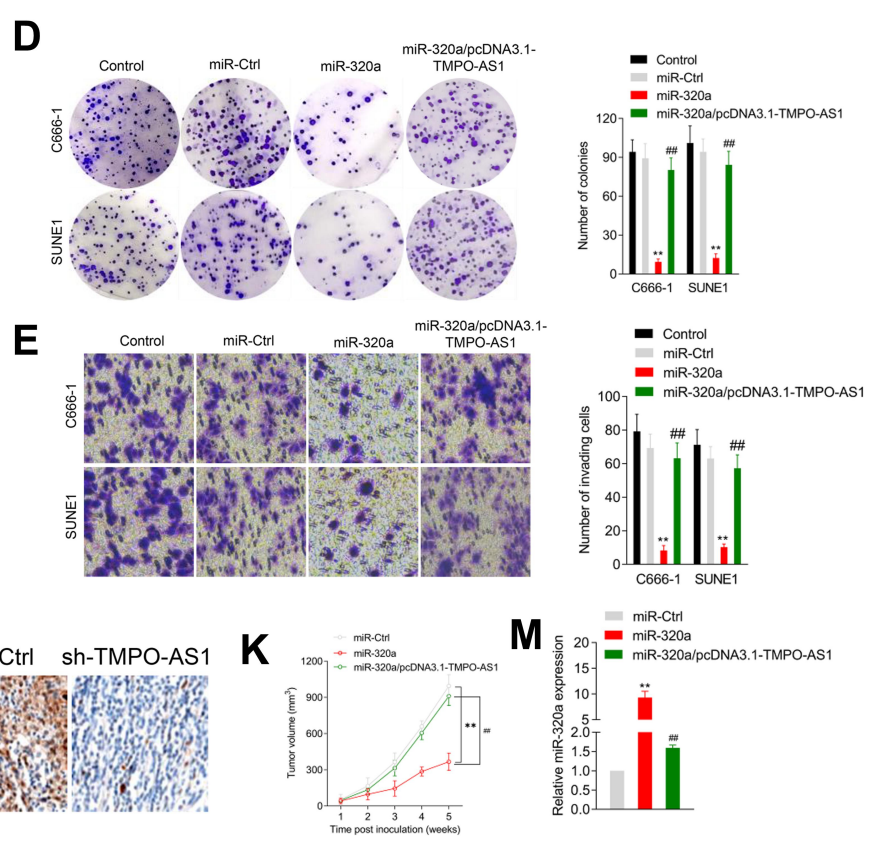

$\mathbf{L}$

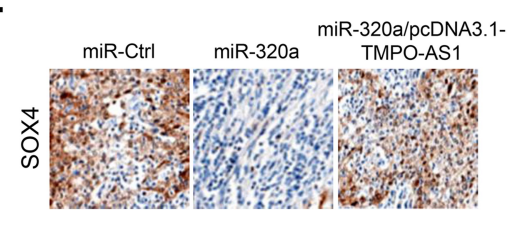

Figure 5 TMPO-ASI regulates the growth and invasion of nasopharyngeal carcinoma cell via sponging miR-320a. (A) C666-I or SUNEI cell was cotransfected with miR320a mimic or miR-320a mimic plus pcDNA3.I-TMPO-ASI. The expression of miR-320a was detected using qRT-PCR assay. (B) The expression of SOX4 was determined by Western blotting analysis. (C) The proliferation of transfected cells was determined by CCK-8 assay. (D and E) Colony formation and Transwell invasion assays were performed with the transfected cells. ${ }^{* * P}<0.01$ compared with miR-Ctrl, ${ }^{\#} P<0.01$ compared with miR-320a. (F) SUNEI cells transfected with sh-Ctrl or sh-TMPO-ASI were subcutaneously injected into the nude mice. The mice were then sacrificed five weeks after the injection. (G) The tumor volumes were measured. (H) The expression of SOX4 in tumor tissue formed by transfected SUNEI cells was determined by immunohistochemical staining. (I) The expression of miR-320a was detected by qRT-PCR. **P<0.0I compared with sh-Ctrl. (J) SUNEI cells transfected with miR-320a mimic or cotransfected with miR-320a and pcDNA-3.I-TMPO-ASI were subcutaneously injected into the nude mice. The mice were then sacrificed five weeks after the injection. (K) The tumor volumes were measured. (L) The expression of SOX4 in tumor tissue formed by transfected SUNEI cells was determined by immunohistochemical staining. (M) The expression of miR-320a was detected by qRT-PCR. $* * P<0.01$ compared with miR-Ctrl, ${ }^{\#} P<0.01$ compared with miR-320a.

by upregulating Erb-B2 Receptor Tyrosine Kinase 2 (ERBB2) via sponging miR-204-3p. ${ }^{25}$ TMPO-AS1 also boosts the migration and invasion of bladder cancer cells via sponging miR-98-5p. ${ }^{26}$ However, knowledge regarding the underlying regulatory mechanism of TMPO-AS1 in NPC remains scarce. The loss-of-function assay illustrated that TMPO-AS1 silencing substantially repressed the proliferation, colony formation, and invasion abilities of NPC cells in vitro. Nevertheless, upregulation of TMPO-AS1 produced opposite results. Additionally, TMPO-AS1 suppression decreased the tumor growth of NPC cells in vivo. Altogether, our findings elucidated the oncogenic action of TMPO-AS1 in cell growth and invasion of NPC.

Functional IncRNAs have been reported to act as a sponge for miRNAs and modulate the expression of target genes at the post-transcriptional level. ${ }^{27}$ Therefore, we speculated that TMPO-AS1 might contribute to NPC cells' proliferation and invasion in a similar manner. In this study, we demonstrated that TMPO-AS1 interacted with miR-320a in NPC cells, using bioinformatics analysis and luciferase reporter assay. Importantly, the level of miR-320a in NPC cells was found to be negatively regulated by TMPO-AS1, as demonstrated by qRT-PCR. Previous studies have revealed that miR-320a acts as an essential suppressor in tumor cell proliferation and metastasis by targeting oncogenes or cancer suppressors. ${ }^{15,28}$ Consistently, we found that miR-320a was abnormally downregulated in NPC. Upregulation of miR-320a remarkably weakened the growth and decreased the colony formation and invasion abilities of NPC cells. These observations indicate that miR-320a has an inhibitory action on the aggressive capabilities of NPC cells. 
miRNAs regulate mRNA stability and/or inhibit mRNA translation by binding the complementary sequences in their target gene's $3^{\prime}$-UTR. ${ }^{1,29}$ Of note, SOX4 was established as a target of miR-320a in NPC. Importantly, TMPO-AS1 silencing and upregulation of miR-320a conspicuously reduced the protein expression of SOX4 in NPC cells. Dysregulation of the ceRNA regulatory axis is involved in the malignant progression of cancers. Herein, we proved that TMPO-AS1 is also a ceRNA that concurrently sponges miR-320a and upregulates its target gene, SOX4. Furthermore, the rescue assays delineated that TMPO-AS1 upregulation could rescue the miR-320a-mediated suppressive effect on cell proliferation and invasion in NPC cells. Similarly, the inhibition of NPC cells' growth in vivo induced by miR320a was distinctly counteracted by TMPO-AS1, suggesting TMPO-AS1 regulated NPC cells' growth in vivo by sponging miR-320a. An early study published previously demonstrated that the upregulation of SOX4 is associated with growth and metastasis in NPC cells. ${ }^{30}$ In addition, SOX4 contributes to transforming growth factor $\beta$ (TGF$\beta)$-induced epithelial-mesenchymal transition (EMT) and stem cell characteristics of gastric cancer cells. ${ }^{31}$ The present study has several limitations. First, the number of clinical samples was small. Prospective studies with larger sample sizes are required to verify the function of TMPOAS1 in NPC. It might be better to investigate the mechanism of lncRNA TMPO-AS1/miR-320a/SOX4 in regulating the EMT of NPC cells.

In conclusion, our work revealed that TMPO-AS1 promoted NPC cells' growth and invasion by sponging miR-320a to elevate SOX4 expression, highlighting TMPOAS1 as a valuable potential therapeutic target for NPC.

\section{Disclosure}

The authors declare that they have no competing interests in this work.

\section{References}

1. Huang Q, Hou S, Zhu X, Liu S. MicroRNA-192 promotes the development of nasopharyngeal carcinoma through targeting RB1 and activating PI3K/AKT pathway. World J Surg Oncol. 2020;18(1):29. doi:10.1186/s12957-020-1798-y

2. Duan B, Shi S, Yue H, et al. Exosomal miR-17-5p promotes angiogenesis in nasopharyngeal carcinoma via targeting BAMBI. $J$ Cancer. 2019;10(26):6681-6692. doi:10.7150/jca.30757

3. Wu S, Xie DL, Dai XY. Down-regulation of miR-155 promotes apoptosis of nasopharyngeal carcinoma CNE-1 cells by targeting PI3K/AKT-FOXO3a signaling. Eur Rev Med Pharmacol Sci. 2019;23(17):7391-7398. doi:10.26355/eurrev_201909_18847
4. Ji L, Fan X, Zhou F, et al. IncRNA RPL34-AS1 inhibits cell proliferation and invasion while promoting apoptosis by competitively binding miR-3663-3p/RGS4 in papillary thyroid cancer. $J$ Cell Physiol. 2020;235(4):3669-3678. doi:10.1002/jcp.29256

5. Wang Y, Bhandari A, Niu J, et al. The lncRNA UNC5B-AS1 promotes proliferation, migration, and invasion in papillary thyroid cancer cell lines. Hum Cell. 2019;32(3):334-342. doi:10.1007/ s13577-019-00242-8

6. Jia Y, Duan Y, Liu T, et al. LncRNA TTN-AS1 promotes migration, invasion, and epithelial mesenchymal transition of lung adenocarcinoma via sponging miR-142-5p to regulate CDK5. Cell Death Dis. 2019;10(8):573. doi:10.1038/s41419-019-1811-y

7. Yang J, Liang B, Hou S. TMPO-AS1 promotes cervical cancer progression by upregulating RAB14 via sponging miR-577. J Gene Med. 2019;21(11):e3125. doi:10.1002/jgm.3125

8. Gang X, Yuan M, Zhang J. Long non-coding RNA TMPO-AS1 promotes cervical cancer cell proliferation, migration, and invasion by regulating miR-143-3p/ZEB1 axis. Cancer Manag Res. 2020;12:1587-1599. doi:10.2147/CMAR.S226409

9. Guo X, Wang Y. LncRNA TMPO-AS1 promotes hepatocellular carcinoma cell proliferation, migration and invasion through sponging miR-329-3p to stimulate FOXK1-mediated AKT/mTOR signaling pathway. Cancer Med. 2020;9(14):5235-5246. doi:10.1002/ cam4.3046

10. Zheng Z, Li X, You H, et al. LncRNA SOCS2-AS1 inhibits progression and metastasis of colorectal cancer through stabilizing SOCS2 and sponging miR-1264. Aging (Albany NY). 2020;12(11): 10517-10526. doi:10.18632/aging.103276

11. Cui H, Zhao J. LncRNA TMPO-AS1 serves as a ceRNA to promote osteosarcoma tumorigenesis by regulating miR-199a-5p/WNT7B axis. J Cell Biochem. 2020;121(3):2284-2293. doi:10.1002/jcb.29451

12. Sun S, Hang T, Zhang B, et al. miRNA-708 functions as a tumor suppressor in colorectal cancer by targeting ZEB1 through Akt/ mTOR signaling pathway. Am J Transl Res. 2019;11(9):5338-5356.

13. Li Z, Rana TM. Therapeutic targeting of microRNAs: current status and future challenges. Nat Rev Drug Discov. 2014;13(8):622-638. doi: $10.1038 / \mathrm{nrd} 4359$

14. van Rooij E, Olson EN. MicroRNA therapeutics for cardiovascular disease: opportunities and obstacles. Nat Rev Drug Discov. 2012;11 (11):860-872. doi:10.1038/nrd3864

15. Li Y, Liu H, Shao J, Xing G. miR-320a serves as a negative regulator in the progression of gastric cancer by targeting RAB14. Mol Med Rep. 2017;16(3):2652-2658. doi:10.3892/mmr.2017.6937

16. Zhao W, Sun Q, Yu Z, et al. MiR-320a-3p/ELF3 axis regulates cell metastasis and invasion in non-small cell lung cancer via PI3K/Akt pathway. Gene. 2018;670:31-37. doi:10.1016/j.gene.2018.05.100

17. Lee HM, Okuda KS, Gonzalez FE, Patel V. Current perspectives on nasopharyngeal carcinoma. Adv Exp Med Biol. 2019;1164:11-34.

18. Lee AWM, Ng WT, Chan JYW, et al. Management of locally recurrent nasopharyngeal carcinoma. Cancer Treat Rev. 2019;79:101890. doi:10.1016/j.ctrv.2019.101890

19. Zhou DN, Ye CS, Yang QQ, Deng YF. Integrated analysis of transcriptome profiling predicts potential lncRNA and circRNA targets in human nasopharyngeal carcinoma. Oncol Lett. 2020;19 (4):3123-3136. doi:10.3892/ol.2020.11412

20. Yang B, Jia L, Ren H, et al. LncRNA DLX6-AS1 increases the expression of HIF-1alpha and promotes the malignant phenotypes of nasopharyngeal carcinoma cells via targeting MiR-199a-5p. Mol Genet Genomic Med. 2020;8(1):e1017. doi:10.1002/mgg3.1017

21. Chen X, Huang Y, Shi D, et al. LncRNA ZNF667-AS1 promotes ABLIM1 expression by adsorbing micro RNA-1290 to suppress nasopharyngeal carcinoma cell progression. Onco Targets Ther. 2020;13:4397-4409. doi:10.2147/OTT.S245554

22. Li Z, Feng Y, Zhang Z, et al. TMPO-AS1 promotes cell proliferation of thyroid cancer via sponging miR-498 to modulate TMPO. Cancer Cell Int. 2020;20(1):294. doi:10.1186/s12935-020-01334-4 
23. Mitobe Y, Ikeda K, Sato W, et al. Proliferation-associated long noncoding RNA, TMPO-AS1, is a potential therapeutic target for triple-negative breast cancer. Cancer Sci. 2020;111(7):2440-2450. doi: $10.1111 /$ cas. 14498

24. Sun Y, Han C. Long non-coding RNA TMPO-AS1 promotes cell migration and invasion by sponging miR-140-5p and inducing SOX4-mediated EMT in gastric cancer. Cancer Manag Res. 2020;12:1261-1268. doi:10.2147/CMAR.S235898

25. Yu X, Lin Q, Liu F, et al. LncRNA TMPO-AS1 facilitates the proliferation and metastasis of NSCLC cells by up-regulating ERBB2 via sponging miR-204-3p. Int J Immunopathol Pharmacol. 2020;34:2058738420958947. doi:10.1177/2058738420958947

26. Luo H, Yang L, Liu C, et al. TMPO-AS1/miR-98-5p/EBF1 feedback loop contributes to the progression of bladder cancer. Int J Biochem Cell Biol. 2020;122:105702. doi:10.1016/j.biocel.2020.105702

27. Tian X, Liu Y, Wang Z, Wu S. IncRNA SNHG8 promotes aggressive behaviors of nasopharyngeal carcinoma via regulating miR-656-3p/ SATB1 axis. Biomed Pharmacother. 2020;131:110564. doi:10.1016/j. biopha.2020.110564
28. Zhao H, Dong T, Zhou H, et al. miR-320a suppresses colorectal cancer progression by targeting Rac1. Carcinogenesis. 2014;35 (4):886-895. doi:10.1093/carcin/bgt378

29. He W, Huang Y, Jiang CC, et al. miR-100 inhibits cell growth and proliferation by targeting HOXA1 in nasopharyngeal carcinoma. Onco Targets Ther. 2020;13:593-602. doi:10.2147/OTT.S228783

30. Shi S, Cao X, Gu M, et al. Upregulated expression of SOX4 is associated with tumor growth and metastasis in nasopharyngeal carcinoma. Dis Markers. 2015;2015:658141. doi:10.1155/2015/ 658141

31. Peng X, Liu G, Peng H, et al. SOX4 contributes to TGF-beta-induced epithelial-mesenchymal transition and stem cell characteristics of gastric cancer cells. Genes Dis. 2018;5(1):49-61. doi:10.1016/j. gendis.2017.12.005

\section{Publish your work in this journal}

Cancer Management and Research is an international, peer-reviewed open access journal focusing on cancer research and the optimal use of preventative and integrated treatment interventions to achieve improved outcomes, enhanced survival and quality of life for the cancer patient.
The manuscript management system is completely online and includes a very quick and fair peer-review system, which is all easy to use. Visit http://www.dovepress.com/testimonials.php to read real quotes from published authors. 